\title{
Individual Knowledge Management with MailTack
}

\author{
Marco C. Bettoni \\ Basel University of Applied Sciences (FHBB) \\ m.bettoni@fhbb.ch \\ Robert Ottiger \\ IKM Lab \\ http://www.ikm-lab.ch/ \\ Rolf Todesco \\ IKM Lab \\ http://www.ikm-lab.ch/ \\ Kurt Zwimpfer \\ IKM Lab \\ http://www.ikm-lab.ch/
}

\begin{abstract}
We present our EU-sponsored research in the domain of individual knowledge management and collaborative learning: the MailTack system. MailTack is a stand-alone software tool that we use to understand and support our behaviour in complex e-mail discussions with the aim of contributing to a systematic Individual Knowledge Management and Team Learning. Based on a constructivist understanding of knowing, we have developed MailTack as a tool for Persistent Business Discussions combined with an Individual Knowledge Assessment. By helping the involved individuals in improving the effectiveness and efficiency of their asynchronous conversations we think that MailTack could contribute to make collaborative learning and asynchronous interactions (for cooperating, coaching, tutoring, etc.) more successful and attractive thus facilitating the dynamics of Communities of Practice and more generally of learning communities.
\end{abstract}

\section{Introduction}

MailTack has been developed in two projects [3]: the Know-Net project [10], part of an Esprit call, started 1998 and finished 2000 and the KnowPort project, a research project from our Individual Knowledge Management Laboratory that is still going on [2].

We begin with a short overview over the result of the Know-Net project to demonstrate the difference and the relation between organisational and individual knowledge management. We will then point out the main goals of the KnowPort project, sketch the basic research question addressed with MailTack and explain some of the key elements of our theoretical background called Radical Constructivism.

On this basis we will then present the characteristics of MailTack and demonstrate its functions and main user interfaces with a screenshot.
Finally we will close by summarising the individual knowledge management capabilities of MailTack.

\section{The Know-Net Approach}

The Know-Net consortium - a group of 8 european companies and academic organisations - cooperated in the development and evaluation of the Know-Net method and tool. The three main goals of the project were to support organisations, teams and individuals in developing their learning capabilities through a systematic knowledge management. Our team at the Basel University of Applied Sciences (FHBB) focused explicitly on the individual learning domain. As mentioned before we would like to give you as first an idea of the tool for supporting the organisational knowledge management [10] in order to distinguish it clearly from our own approach.

The Know-Net tool is based on a Lotus Domino Server and includes a relational database with all the knowledge objects. On top of this there is a process and application level and finally a navigators level with three different user interfaces to the two lower levels: a System Administrator Navigator, a Strategic Knowledge Navigator and a Knowledge Worker Navigator.

Now let us have a look at the strategic navigator. A socalled knowledge broker or officer works with the strategic navigator. She defines all the knowledge assets that build the core around which the organisation's knowledge is systematically managed. Then she defines for the entire organisation - knowledge objects for the knowledge strategy, the processes, the systems and the structures. Finally she defines knowledge objects for networking knowledge flows at 4 different organisational levels: individual, team, organisational and interorganisational level.

The third user interface is the so-called Knowledge Worker Navigator. In Know-Net the knowledge worker is conceived as a kind of "interpreter": he or she has to "play the keyboard" and all he can play is predefined by

\footnotetext{
${ }^{1}$ Accepted for Third Intern. Workshop on Theory and Applications of Knowledge MAnagement as part of DEXA 2002
} 
the strategic knowledge officer or - in other words - by the "composer". Of course the knowledge worker can define his meetings, can describe her lessons learned and see the lessons that other workers have learned, but besides learning to use the tool the knowledge worker does not use the tool to learn, but to work like a trivial machine. We may say, that the learning subject in this setting is not the knowledge worker, but the organisation.

But can an organisation manage its knowledge processes and learn in a sustainable way without promoting the ability of its individuals to learn and to manage their own, individual knowledge? We are convinced that the answer is no! Our conception of a more appropriate relation between organisational and individual knowledge management is under development in two ongoing research projects, the first called KnowPort [2] and the second called Vico \& Co [4].

\section{The KnowPort Approach}

The KnowPort approach starts by claiming that as soon as individuals begin working with a knowledge management infrastructure for managing (sharing etc.) knowledge within their teams and enterprises, a profound cultural change in their competency requirements, roles and responsibilities takes place. The objective of the KnowPort project is to support these individuals in meeting the challenge of such a cultural change by helping them in the task of self-managing their own knowledge resources. To this aim, in the KnowPort project we construct tools that address the need implicit in the sentence: "If I only knew what I know!". One of our visions is that each person can compose an individual knowledge portfolio using the so-called "Trace your Tack" principle, which states:

"For sailing on the right tack in the knowledge ocean and mastering the information overflow by entering safely new ports of knowledge trace your knowledge in action (on tack)" [2, 6-2].

We all work in a very specific or individual way using different methods and tools to achieve our goals. If we try to understand what we are doing, then tracing our tack while sailing in the knowledge ocean can provide a valuable support by means of which we can become aware of what we know and of where we would like to invest to become more efficient and effective.

MailTack is only a part of our toolset that we plan to construct. MailTack was chosen to start with because we where unsatisfied with our efficiency and effectiveness in complex mail discussions in which we were discussing our ideas for improving the discipline of knowledge management $[3,11]$. Our basic research question was if and how it would be possible to support collaborating individuals in becoming more efficient and effective in "knowledge work" by enhancing the visibility and transparency of the knowledge that they apply or develop in working, learning and sharing.
For identifying a relevant kind of support we considered that when managing knowledge work, a central issue consists in figuring out what the individual already knows: for this reason the first research objective was to allow the user to make an Individual Knowledge Assessment (IKA) on a continuous basis as an implementation of the above mentioned "trace your tack" principle.

We further considered that when doing knowledge work, two salient properties are:

1. communication with other professionals

2. the ability and willingness to share knowledge.

Both skills can be greatly improved and developed if the individual engages in conversations (discussions): for this reason the second research objective was to allow the user to do persistent asynchronous conversations in a sustainable way.

Our conception of the MailTack tool has been influenced by related work done by A. Abecker at DFKI [1], J. Donath at MIT [6] and by Thomas Erickson at IBM Watson Research Center [7]. They show among other things, that when conversation takes place as computermediated conversation (CMC) it becomes cumbersome, particularly among more participants, lacks transparency, looses coherence tending towards drift and dissolution after few interactions: this kind of conversation is not sustainable as we all know (but seem to ignore)!

Software tools that have been a source of inspiration include the 'KARAT' tool by DFKI and 'The Brain' by Natrificial LLC which both should enable its user to "use your computer the way you use your mind - intuitively". 'Knowledge $\mathrm{X}$ ' by IBM, which like MailTack uses knowledge maps to represent concepts and their relationships, was delivered when our tool had already been designed (June '99) and encouraged us to continue in the selected direction.

\section{Radical Constructivism}

One fundamental claim that we share with Radical Constructivism is that observers are operationally closed systems. Every person lives in its own experiential world. You, we, our colleagues, everybody in the real sense of the word have to create a coherent, consistent and viable system of connected viable mental operands (his experiential world) using nothing else then the capabilities of our own body. With our bodies we make the experiences that our bodies allow. We behave - as Humberto Maturana says - like a captain in a submarine, having nothing else to observe than the state of the submarine (or body) using the states of the instruments (or sensors) available [9]. To put it in other words, we cannot compare our experiences with something out there (the real, the truth) but only with other own experiences. This sight was called radical by one of the pioneers of constructivism, Ernst von Glasersfeld who, in this context, also developed the concept of viability [8]. 
Another fundamental claim is that we consider explanations as manufactured mechanisms. This point is a consequence of taking the term "construction" seriously. We constitute our own experiential world (the system of viable mental operands previously mentioned) but we manufacture the Eiffel Tower as an artefact by transforming (handling) physical objects. What we exactly do, when we constitute our own world, we cannot analyse in the same way as we can analyse the manufacturing of the Eiffel Tower or - to take a more complex example - the manufacturing of the computer that we use here to type this paper. We only can understand a thing in the sense of "begreifen" ("auf den Begriff bringen") if we manufactured it or in other words, we only can understand our artefacts.

If you followed the argumentation so far, the next consequence is that the discipline dealing with manufacturing artefacts (physical constructions) becomes the basis of our understanding. Science in this sense does not look for the truth, but for viable explanations.

\section{The MailTack System}

When we work with MailTack we construct a knowledge base (MailKB) that is structured with the following elements (knowledge schema):

- Sentence: elementary conversation unit (conceptual element, does not appear as a system element in the MailKB)

- Segment: connection of adjacent expressions in a text

- Category: concept (keyword, expression) for designating thematic views

- Stream: connection of segments (from one or different texts) into a sequence

- Constellation: association of categories with other categories in a flat network

- Categorization: association of segments with categories of a constellation

If you remember the formerly mentioned knowledge assets defined by the knowledge officer on the strategic level in the Know-Net example (par. 2) you will find here a parallel situation: we, as the constructors of the tool, define the invariants (knowledge schema) that we, as users of the tool, cannot change.

\subsection{Using MailTack's knowledge schema}

According to the given knowledge schema, the user who wants to work with MailTack must organize the network of her e-mail conversations as follows: unite message sentences into segments, define categories and connect them into constellations, associate segments with categories and connect segments into conversation streams. Segments and categories are the building bricks of a MailKB. Segments are created from given e-mail messages whereas categories are (mainly) created from existing categories. Through connections and associations every segment and every category is directly or indirectly related to every other segment or category. Thus MailTack lets you create a kind of knowledge base that is highly interconnected, quite like knowledge in your mind. A MailKB organizes e-mail messages into a highly personal conceptual space. By organizing your MailKB by your subject matters in associated areas, when you are thinking about a particular subject or idea MailTack will present you segments that contain that subject or idea with their context. You will then be able to quickly focus your attention and contribute more efficiently and more effectively new ideas to an ongoing e-mail conversation.

Just for those who are interested in more technical details some constructive characteristics of MailTack:

- Local PC Client Application

- MIME (Multipurpose Internet Mail Extensions)

- Text Segmentation (ASCII)

- Mind Mapping and Semantic Networks (GUI)

- Relational Database (ODBC)

\subsection{A session with MailTack}

Now let us consider the user interface in more details in order to get an idea of how a MailTack session looks like. After starting the tool an MDI window collecting 4 windows appears and the most recent MailKB is loaded. This is called 'Desktop A' and is composed by the windows 'New Messages', 'All Mails', 'Constellations' and 'All Categories'. The first window 'New Messages' is a list of unread e-mails: simply clicking on one list item opens the message in a viewer. After a quick look the user can decide about its relevance and import it into the MailKB by one click: a new entry then appears in the window 'All Mails', a list of imported messages below the first window. On the right upper side a graph of categories and their connections is displayed in the 'Constellations' window and finally below this the 'All Categories' window displays the same categories as alphabetic list.

Selecting 'Desktop B' provides an environment in which the content of relevant messages can be segmented and categorized. In this desktop the window 'All Mails' is in the upper left position. Clicking on an item opens the message in the editor (lower left corner). Here we can do the segmentation and categorisation: mark a piece of text, specify it as 'segment' by one click and associate it with categories. The segment with its categorizations appears in the 'Categorisations' window (a graph editor on the right upper corner) as a box surrounded by categories and connected with each of them like in a semantic net. Clicking on the segment box displays its text in the text window 'Segments A \& B' below (lower left corner).

When the relevant message text has been segmented and the segments categorized, then all the new segments can be linked together and with other segments from previous messages by working in desktop C (see Fig.1). 
The 3 windows 'Categorizations' (up left), 'Constellations' (up right) and 'Segments A \& B' (low right) were already either in desktop A or B: the window called 'Streams' (low left) instead is new. This is a graph editor displaying argumentation threads as networks of boxes. The user creates these networks as follows: when a new segment is created it appears in this graph editor as an unconnected numbered box (for example box 17 in Fig.1); selecting it together with a second box (box 6) allows to create a directed connection between the two. During this operation the text of the first and second segment appears in the viewer on the right side.

To summarise, let us have a look at the basic elements of MailTack. The user is supported in doing the following tasks: importing relevant mail from his or her standard mail tool, defining relevant text segments, associating segments with categories (categorisations), associating categories with other categories (constellations) and finally associating segments into discussion threads (streams). All the data related to these knowledge structures are stored as tables of a relational database. Between the user interface functions and the database we have an instance layer being served with a load/save mechanism. For handling the elements of the MailKB the main work is done manually by the user through the GUI: but we have planned to automate part of this manual work by means of rule based inferences implemented with Aion, a rule based development environment [5]. The graphical user interface is driven by an ActiveX tool from Tom Sawyer, the database is managed with FoxPro and finally the shell with all the different windows is developed in VisualBasic.

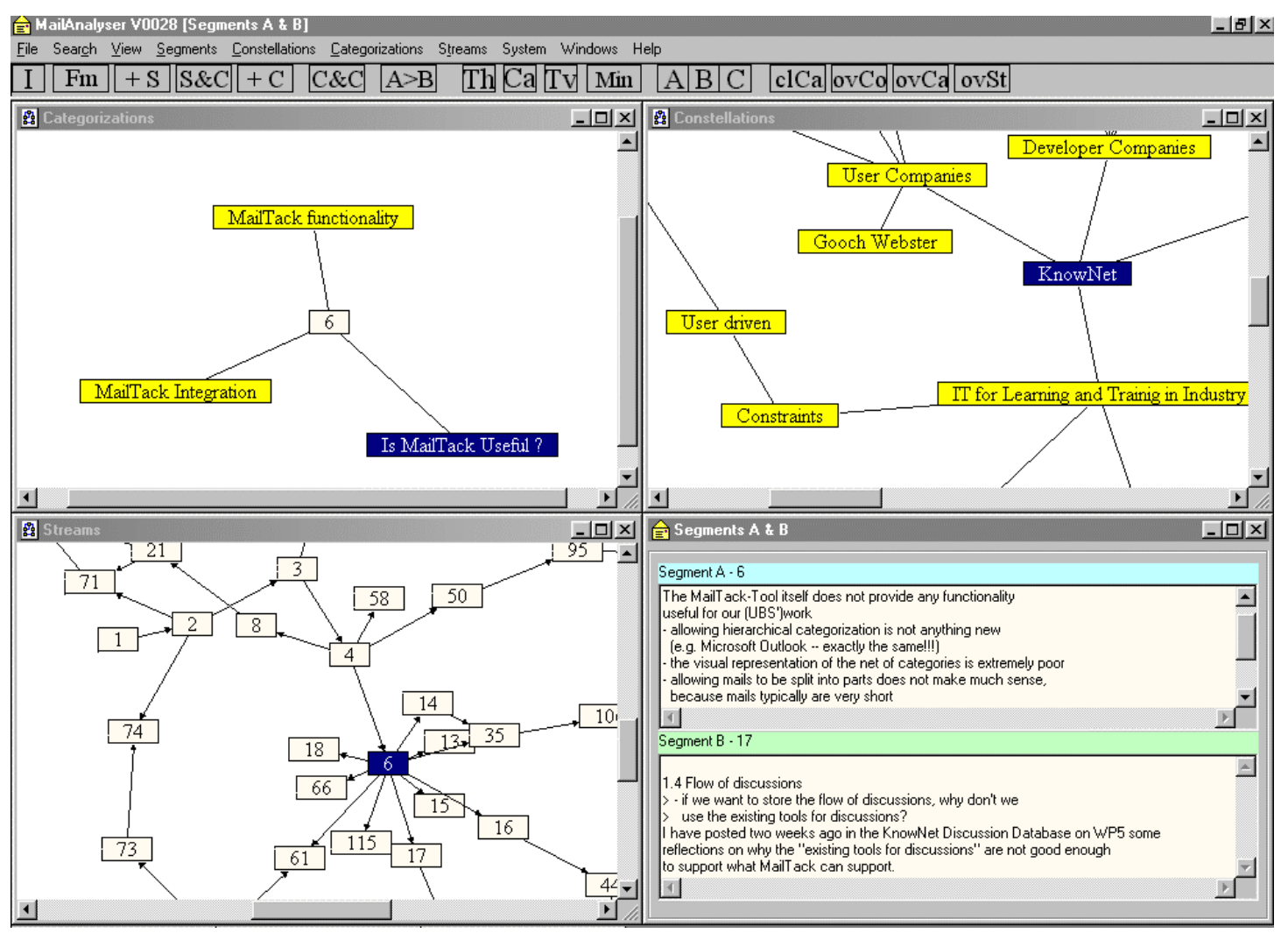

Fig.1: MailTack Desktop C

\section{Conclusion: Can we use MailTack?}

Taking seriously the observation that "It is through conversation that we create, develop, validate and share knowledge" (T. Erickson) we have developed a tool for Persistent Business Discussions combined with an Individual Knowledge Assessment that promotes such skills as:

- $\quad$ accumulating relevant knowledge bits
- $\quad$ accessing those bits of knowledge, much of which one does not immediately remember

- reusing what I know and sharing what I know

- ensuring that what I say is interpreted correctly

- negotiating and structuring controversy

- mapping and storing knowledge in a more manageable way

By helping in this way the involved individuals in improving the effectiveness and efficiency of their asynchronous conversations we think that MailTack could 
contribute in making individual knowledge management, asynchronous knowledge sharing in teams and collaborative learning more successful and attractive.

The 3 main lessons we learned as far from the development and use of MailTack can be summarised as follows:

1. We have learned that tracing the tack in complex email conversations is a big challenge: up to now all our MailKB's still have an experimental character. Using MailTack, e-mails become a domain of competence requiring and promoting the skills previously mentioned. Being able to use the tool in the sense of applying it technically we realised that the intellectual demand to build a MailKB is a big effort that we normally cannot afford in daily business mail conversation: in a certain sense it is like building and steadily dynamically adapting an individual "ontology". To support this effort we are planning to develop a specific methodology inspired by the work on organizational ontologies but adapted to fit with our radical constructivist foundation (par. 4) and our focus on balancing the individual and collective perspective.

2. Constellations and streams became an important concept in our discussions in the domain of knowledge management and learning.

3. Having built the tool as an artefact we use it as a base of meta-knowledge to explain our behaviour in a constructive way.

\section{References}

[1] Abecker A., Schmalhofer F. and Tschaitschian B., Information Tuning with KARAT: Capitalizing on Existing Documents. In: E. Plaza and R. Benjamins (eds.), $10^{\text {th }}$ European Workshop on Knowledge Acquisition, Modeling, and Management (EKAW-97). Sant Feliu de Guíxols, Catalonia, Spain. Springer Verlag, LNAI 1319, October 1997.
[2] Bettoni M. Ottiger, R., Todesco, R. and Zwimpfer, K., KnowPort - A Personal Knowledge Portfolio Tool, Proceedings of PAKM 98, 2nd Int. Conf. on Practical Aspects of Knowledge Management, 29 - 30 October 1998, Basel.

[3] Bettoni, M., Ottiger, R., Todesco, R. and Zwimpfer, K., Learning by e-Conversation with MailTack, Online Educa Berlin $-7^{\text {th }}$ Int. Conf. on Technology Supported Learning \& Training, 28 - 30 November, 2001.

[4] Bettoni, M. and Schneider, S., Experience Management: Lessons Learned from Knowledge Engineering, In: M. Minor, S.Staab, $1^{\text {st }}$ German Workshop on Experience Management, Lecture Notes in Informatics (LNI) Vol P-10, Gesellschaft für Informatik (GI), Bonn, 2002, ISBN 3-88579-340-7, pp. 117-128

[5] Aion Product Information (marketing and customer stories): http://www.ca.com/products/aion/aion_pd.htm

[6] Donath J. et. al., Visualizing Conversation, J. of ComputerMediated Communication, 4 (4), June 1999.

[7] Erickson T. and Kellog W., Social Translucence: An Approach to Designing Systems that Support Social Processes, Transactions on Computer Human Interface, ACM Press, 2000.

[8] von Glasersfeld, E.: Radical Constructivism: A Way of Knowing and Learning, Falmer Press, London, 1996.

[9] Maturana, H., Erkennen: Die Organisation und Verkörperung von Wirklichkeit. Vieweg, Braunschweig, 1982.

[10] Mentzas G, Apostolou D., Young R. and Abecker A.: Knowledge Networking: A Holistic Approach, Method and Tool for Leveraging Corporate Knowledge. Journal of Knowledge Management. 5(1): 94-106, MCB University Press, 2001.

[11] Todesco, R. - MailTack: Individuelles Wissensmanagement, in: Handler P et al., (Eds.): Textproduktion. Proceedings of the $4^{\text {th }}$ Prowitec-Kolloquium, Wien, April 2000 (in press 2001). 